\title{
Coronary sinus aneurysm associated with multiple venous anomalies
}

\author{
Guang Song ${ }^{1}$, Ming Du', Weidong Ren ${ }^{1 *}$ (D) Ke Zhou ${ }^{3}$ and Lu Sun ${ }^{1}$
}

\begin{abstract}
Background: Congenital anomalies of the venous system are rare, involve the inferior vena cava (IVC), a persistent left superior vena cava (PLSVC), and the left hepatic vein (LHV), and can make cardiac diagnostic and therapeutic procedures difficult.

Case presentation: We present a 67-year-old woman without heterotaxy syndrome associated with interruption of the left IVC that continued with the hemiazygos vein system, a PLSVC, and an anomalous LHV draining the into coronary sinus (CS). The venous anomalies caused a CS aneurysm. The anomalies were demonstrated by echocardiography and the diagnosis was established by contrast-enhanced computed tomography. Three days later, a coronary artery bypass graft was performed, which confirmed the diagnosis. Half a month after surgery, the pain had been relieved and the patient was discharged from the hospital.

Conclusion: Echocardiography is a useful modality to diagnose and assess anomalies of the CS, including CS aneurysms. Congenital anomalies of the venous system in this case were all due to embryonic development abnormalities. Contrast-enhanced computed tomography provides a more comprehensive view of the entire course of abnormal veins.
\end{abstract}

Keywords: Interruption, Left inferior vena cava, Persistent left superior vena cava, Hepatic vein, Hemiazygos vein, Coronary sinus, Aneurysm

\section{Background}

Congenital anomalies of the inferior vena cava (IVC) are rare, with a prevalence of $1 \%$, and include interruption of the IVC, left IVC, and double IVC. Interruption of the IVC is a well-recognized, but uncommon anatomic anomaly [1]. The most common form of this anomaly is a right IVC that continues with the azygos vein via the right superior vena cava (R-SVC) into the right atrium (RA). A left IVC is also rare. When a left IVC is interrupted, the condition is more complicated because there are numerous routes for the return of blood [2]. In addition, interruption of the IVC is often associated with heterotaxy syndrome. If a patient does not have other features of the heterotaxy syndrome, it is easy for the examiner to miss the diagnosis. Herein, we present a patient without heterotaxy syndrome associated with interruption of the left IVC that continued with the

\footnotetext{
* Correspondence: renwdcmu@163.com

${ }^{1}$ Department of Ultrasound, Shengjing Hospital of China Medical University,

Shenyang, China

Full list of author information is available at the end of the article
}

hemiazygos vein system, a persistent left superior vena cava (PLSVC), and an anomalous left hepatic vein (LHV) connected with the coronary sinus (CS). Together, the venous anomalies caused a coronary sinus aneurysm (CSA).

\section{Case presentation}

A 67-year-old woman had intermittent chest pain for 1 year. She underwent coronary angiography and was diagnosed with significant coronary artery disease involving three main coronary arteries at the referring hospital. All three main coronary arteries had stenotic changes. She presented to our hospital for a coronary artery bypass graft. At the time of the clinical examination, the pulse rate was 85 beats/min and the blood pressure was 132/84 mmHg. No murmurs were auscultated. The electrocardiogram showed persistent atrial fibrillation with abnormal ST segment changes. Echocardiography demonstrated a dilated CS (Fig. 1). Thoracic and abdominal contrast-enhanced computed tomography was performed on a 64-detector row scanner (Siemens, 


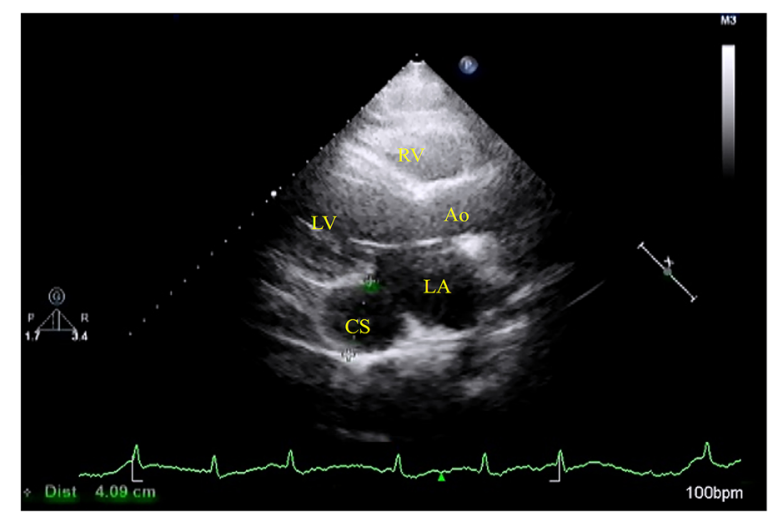

Fig. 1 Parasternal long-axis view from echocardiography demonstrated the coronary sinus aneurysm with a diameter of $4.09 \mathrm{~cm}$. Ao: aorta; CS: coronary sinus; LA: left atrium; LV: left ventricle; RV: right ventricle

Forchheim, Germany). Images were obtained during patient breath-holding using the following acquisition parameters: $120 \mathrm{kV} ; 240 \mathrm{~mA}$; and $1.5-\mathrm{mm}$ thick contiguous section. The patient received $80 \mathrm{~mL}$ of contrast media (Iohexol 350; GE Healthcare, Shanghai, China) using a power injector at $3.5 \mathrm{~mL} / \mathrm{s}$, and the time delays from injection of the contrast agent to scanning were approximately $20 \mathrm{~s}$ and $60 \mathrm{~s}$ for the arterial and venous phases, respectively. Thoracic and abdominal contrastenhanced computed tomography revealed interruption of the left IVC that continued with the hemiazygos vein system, a PLSVC, and an anomalous LHV draining into the CSA (Figs. 2 \& 3; Additional file 1: Movie 1). Considering her advanced age, the physicians did not plan to correct the venous anomalies. Three days later, a coronary artery bypass graft was performed, which confirmed the diagnosis. Half a month after the surgery, the pain was relieved and the patient was discharged from the hospital.

\section{Discussion}

Various diagnostic and therapeutic procedures involving the right side of the heart, such as electrophysiologic studies, right heart catheterization, cardiopulmonary bypass surgery, IVC filter placement, and temporary pacing, have increased the need for ready access to the IVC via the transfemoral route. Anatomic anomalies of the IVC can make these procedures difficult.

The interruption of the IVC has a prevalence of $0.6 \%-$ $2.0 \%$ in patients with congenital heart disease and $0.3 \%$ in healthy people [3]. Embryologically, the normal IVC consists of five segments (hepatic, suprarenal, renal, infrarenal [subrenal], and iliac), which are mainly derived from the right vitelline vein (hepatocardiac canal), the right subcardinal vein, the subcardial and supracardial anastomosis, the supracardial vein, and the persistent posterior cardinal veins. Suprarenal interruption of the IVC in the current case was due to a failed connection between the right vitelline and right subcardinal veins. The blood circulating in the caudal segments of the IVC

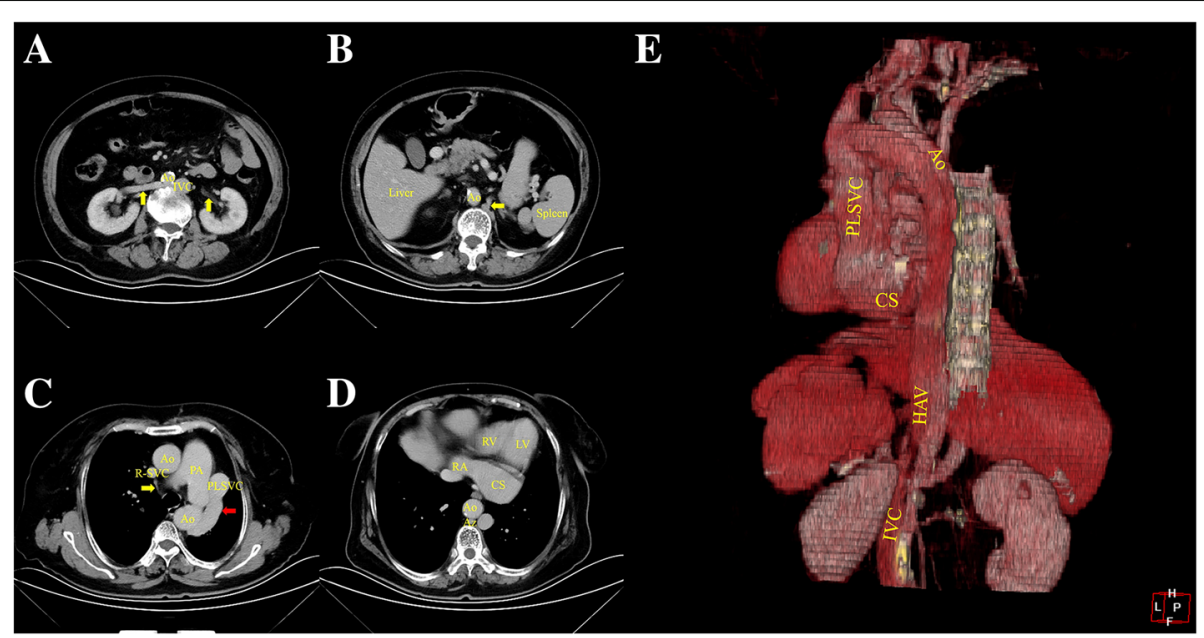

Fig. 2 Thoracic and abdominal enhanced computed tomography demonstrating (a) A left IVC posterolateral to the abdominal aorta, converged by two renal veins (yellow arrow). b The left IVC gradually becomes thin, and continues with an enlarged hemiazygos vein (yellow arrow). Visceral position is normal. c The tiny azygos vein can be seen anterior to the vertebra, then crosses over the aorta and drains into the R-SVC (yellow arrow). The hemiazygos vein continues as the left superior intercostal vein via the accessory hemiazygos vein. The left superior intercostal vein eventually drained into the PLSVC at the level of the pulmonary artery bifurcation (red arrow). $\mathbf{d}$ In the left atrioventricular groove, a huge CS connected with the RA. e Reconstructive 3D imaging gives a better view of this anomaly. Ao: aorta; Az: azygos vein; CS: coronary sinus; HAV: hemiazygos vein; IVC: inferior vena cava; LV: left ventricle; PA: pulmonary artery; PLSVC: persistent left superior vena cava; RA: right atrium; R-SVC: right superior vena cava; RV: right ventricle 


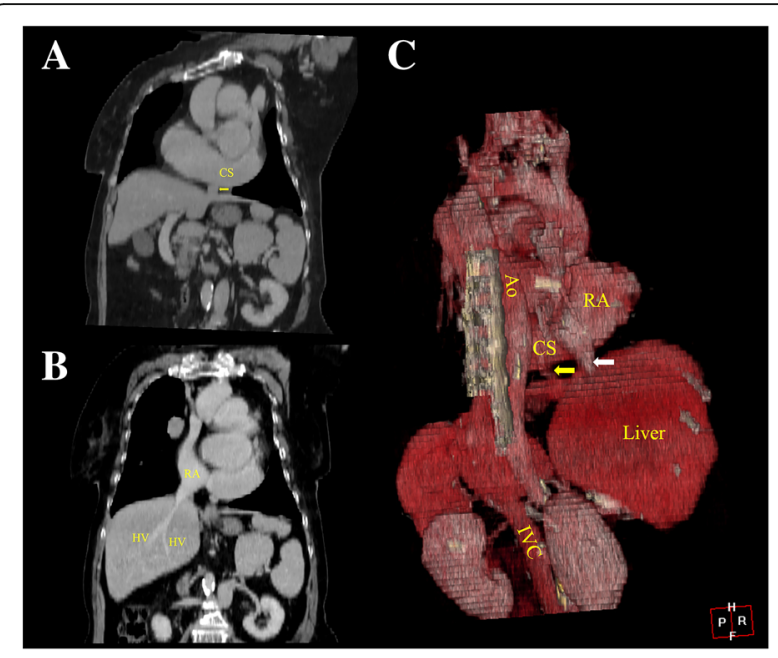

Fig. 3 Thoracic and abdominal enhanced computed tomography demonstrating (a) The left hepatic vein drains into the CS (yellow arrow). b Other hepatic veins drain directly into the right atrium. c Reconstructive 3D imaging revealed that the left hepatic vein drains into the CS (yellow arrow), and other hepatic veins drain directly into the right atrium (white arrow). Ao: aorta; CS: coronary sinus; HV: hepatic vein; IVC: inferior vena cava; LHV: left hepatic vein; PLSVC: persistent left superior vena cava; RA: right atrium

reaches the azygos/hemiazygos system by way of a persistent right/left supracardinal vein. The interruption of the IVC can be associated with cardiac abnormalities, heterotaxy syndrome (polysplenia and asplenia), deep vein thrombosis, and renal vein anomalies, and has also been reported as an asymptomatic incidental finding. Of patients associated with interruption of the IVC without other features of the heterotaxy syndrome, $62.7 \%$ do not have any cardiac malformations, which easily lead to misdiagnosis of this anomaly during echocardiography [4].

The left IVC has a prevalence of $0.2 \%-0.5 \%$, and develops as a result of the persistence of the left supracardinal vein and regression of the right supracardinal vein [5]. Usually, the left IVC crosses over to the right side at the level of the renal veins without interruption. Interruption of the left IVC is rare, and only accounts for $15.5 \%$ of all interruptions involving the IVC [4]. Unlike the interrupted right IVC, which always continues via the azygos vein draining into the R-SVC (97.7\%), the interrupted left IVC continues via a number of routes. Haswell et al. [2] has described three routes for the interrupted left IVC: (1) interrupted left IVC $\rightarrow$ hemiazygos vein $\rightarrow$ azygos vein $\rightarrow$ R-SVC $\rightarrow$ RA; (2) interrupted left IVC $\rightarrow$ hemiazygos vein $\rightarrow$ accessory hemiazygos vein $\rightarrow$ left intercostals vein $\rightarrow$ PLSVC $\rightarrow$ CS $\rightarrow$ RA; and (3) interrupted left IVC $\rightarrow$ hemiazygos vein $\rightarrow$ accessory hemiazygos vein $\rightarrow$ left intercostals vein $\rightarrow$ left brachiocephalic vein $\rightarrow$ R-SVC $\rightarrow$ RA. Subsequently, other routes have been discovered. Based on a review of the literature, five other types of interrupted left IVCs have been described, including the mixed type (Fig. 4) [6-8].

An anomalous LHV connection with the CS has rarely been reported in the literature; indeed, only 14 cases have been reported up to 2015 (Table 1). An anomalous LHV connection with the CS is due to the persistence of the left vitelline connection with the left sinus horn. The diagnosis of this anomaly has important clinical significance. First, an anomalous LHV connection with the CS can potentially lead to technical difficulties during transcoronary sinus interventions and cannulation of the coronary veins for certain procedures, such as biventricular pacing. Second, this anomaly should be detected before cardiac surgery which uses a heart-lung machine because control of venous return will be challenging. Third, this anomaly should be awared in hepatic surgery and transplantation.

In patients with interruption of the IVC, the hepatic veins usually drain directly into the RA. Sometimes, the hepatic veins may connect with a residual IVC that eventually drains into the RA, pulmonary vein, or left atrium [9-11]. Our case is the first report involving multiple systemic vein anomalies.

The above-mentioned multiple venous anomalies involve the vast majority of venous blood draining into the CS. Thus, intimal hyperplasia and loss of smooth muscle and elastic tissue with replacement by connective tissue with age may aggravate the formation of CSA [12]. CSA

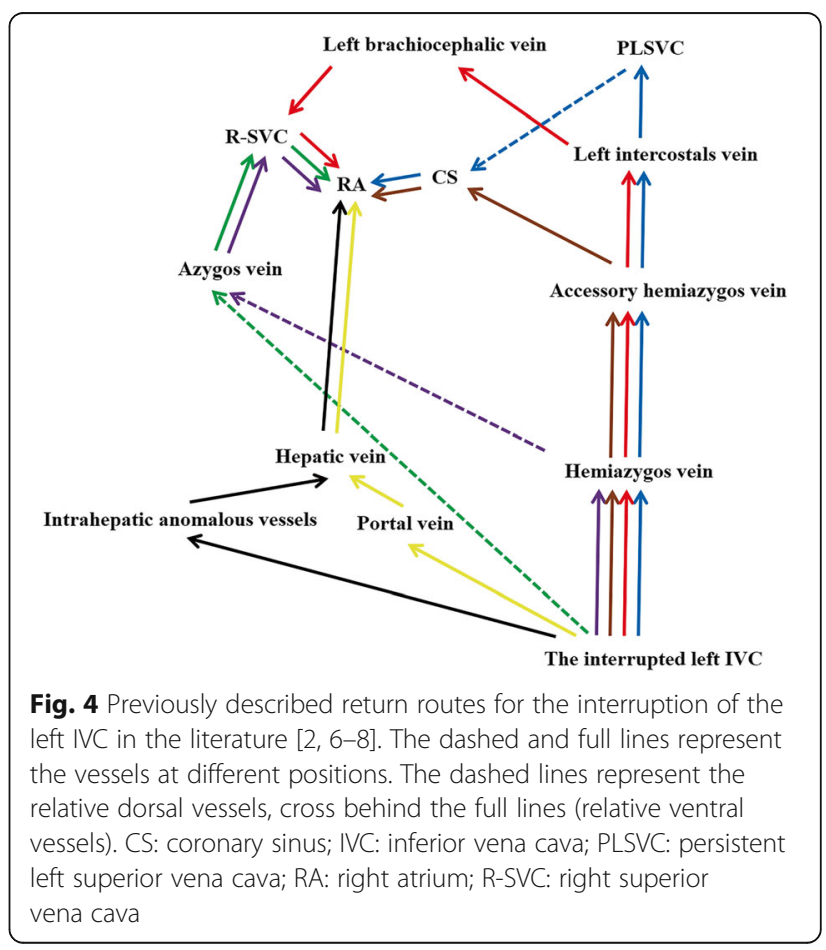


Table 1 Summary of literature involving with anomalous LHV connection with CS

\begin{tabular}{|c|c|c|c|c|c|}
\hline No. & First Author & Year & Sex, age & First modality for diagnosis & Associated cardiovascular anomalies \\
\hline 1 & Nabarro D & 1903 & $\mathrm{M}, 3 \mathrm{~m}$ & Autopsy & PLSVC \\
\hline 2 & Winter FS & 1954 & 1,1 & / & PLSVC \\
\hline 3 & & & 1,1 & / & PLSVC \\
\hline 4 & & & 1,1 & / & PLSVC \\
\hline 5 & van der Horst RL & 1971 & $M, 2 y$ & Angiography & PLSVC, ASD, PS \\
\hline 6 & Bunger PC & 1981 & $F, 90 y$ & Autopsy & PLSVC, atrial fibrillation \\
\hline 7 & Bunger PC & 1982 & $M, 74 y$ & Autopsy & None \\
\hline 8 & Sanders SP & 1984 & $/, 1 \mathrm{~d}$ & Echocardiography & PDA, ASD, ductus venosus to the CS \\
\hline 9 & Mantri RR & 1994 & $F, 12 y$ & Angiography & PLSVC, PS, bifurcation of IVC \\
\hline 10 & Yoshinaga $\mathrm{K}$ & 1997 & $M, 60 y$ & Autopsy & None \\
\hline 11 & Vuran C & 2011 & M, 6y & Intra-operation & ASD, VSD, PDA, pulmonary mass \\
\hline 12 & Buehler M & 2011 & $\mathrm{~F}, 19 \mathrm{y}$ & CT & PLSVC, subaortic valve stenosis, absence of R-SVC \\
\hline 13 & Lee C & 2013 & $F, 61 y$ & CT & None \\
\hline 14 & Morshuis WG & 2015 & $F, 76 y$ & Intra-operation & Three-vessel coronary artery disease \\
\hline
\end{tabular}

$A S D$ atrial septal defect, CSA coronary sinus aneurysm, CT computerized tomography, IVC inferior vena cava, PAPVC partial anomalous pulmonary venous connection, PDA patent ductus arteriosus, PLSVC persistent left superior vena cava, PS pulmonary stenosis, R-SVC right superior vena cava, UCS unroofed coronary sinus, VSD interventricular septal defect

should be diagnosed as early as possible because the CSA in children may compress the mitral annulus and limit atrioventricular flow into the left heart, eventually causing left ventricle, aortic hypoplasia, and obstructive lesions in the left ventricle [13].

\section{Conclusions}

This is the first report involving these three co-existing systemic vein anomalies. Dilated CS by echocardiography is an important diagnostic clue for systemic anomalous venous pathways. Contrast-enhanced computed tomography provides a more comprehensive view of the entire course of abnormal veins. These congenital anomalies can be explained by the theory of embryonic development abnormalities.

\section{Additional file}

Additional file 1: Movie 1. Reconstructive 3D computed tomography demonstrating association with interruption of the left inferior vena cava that continues with the hemiazygos vein system, a persistent left superior vena cava, and an anomalous left hepatic vein draining into the coronary sinus. (AVI 12,902.4 kb)

\section{Abbreviations}

CS: Coronary sinus (CS); CSA: Coronary sinus aneurysm; IVC: Inferior vena cava; LHV: Left hepatic vein; PLSVC: Persistent left superior vena cava; RA: Right atrium; R-SVC: Right superior vena cava

\section{Acknowledgements}

None.

\section{Funding}

None.
Availability of data and material

All data is available in the manuscript.

\section{Authors' contributions}

GS drafted the manuscript and acquired the images. MD acquired the $C$ images. WR acquired the echocardiography images and revised the manuscript. KZ revised the manuscript. LS drafted the manuscript. All authors read and approved the final manuscript. All authors take responsibility for all aspects of the reliability and freedom from bias of the data presented and interpretation

\section{Competing interests}

The authors declare that they have no competing interests.

\section{Consent for publication}

Written informed consent for the publication was obtained from the patient of this case report and any accompanying images and movies.

\section{Ethics approval and consent to participate}

The publication of this case report was in accordance with the Declaration of Helsinki and approved by the ethics committee of Shengjing Hospital of China Medical University.

\section{Publisher's Note}

Springer Nature remains neutral with regard to jurisdictional claims in published maps and institutional affiliations.

\section{Author details}

${ }^{1}$ Department of Ultrasound, Shengjing Hospital of China Medical University, Shenyang, China. ${ }^{2}$ Department of Radiology, Shengjing Hospital of China Medical University, Shenyang, China. 'Department of Cardiac Surgery, Shengjing Hospital of China Medical University, Shenyang, China.

Received: 3 December 2016 Accepted: 1 April 2017

Published online: 05 April 2017

\section{References}

1. Anderson RC, Adams P, Burke B. Anomalous inferior vena cava with azygos continuation (infrahepatic interruption of the inferior vena cava). Report of 15 new cases. J Pediatr. 1961;59:370-83. 
2. Haswell DM, Berrigan TJ. Anomalous inferior vena cava with accessory hemiazygos continuation. Radiology. 1976;119:51-4.

3. Yilmaz E, Gulcu A, Sal S, Obuz F. Interruption of the inferior vena cava with azygos/hemiazygos continuation accompanied by distinct renal vein anomalies: MRA and CT assessment. Abdom Imaging. 2003;28:392-4.

4. Bartram U, Fischer $\mathrm{G}$, Kramer HH. Congenitally interrupted inferior vena cava without other features of the heterotaxy syndrome: report of five cases and characterization of a rare entity. Pediatr Dev Pathol. 2008;11:266-73.

5. Kandpal H, Sharma R, Gamangatti S, Srivastava DN, Vashisht S. Imaging the inferior vena cava: a road less traveled. Radiographics. 2008;28:669-89.

6. Allen HA, Haney PJ. Case report. Left-sided inferior vena cava with hemiazygos continuation. J Comput Assist Tomogr. 1981;5:917-20.

7. Ghossain MA, Haddad SN, Aoun NJ, Atallah NG, Jebara VA, Moussally AS, et al. Portal and hemiazygos continuation of the inferior vena cava: report of an unusual case. Br J Radiol. 1988:61:333-4.

8. Balkanci F, Ozmen MN. Case report: interruption of the inferior vena cava with anomalous intrahepatic continuation. Br J Radiol. 1993;66:457-9.

9. Ihara K, Nitta J, Hirao K, Isobe M. Atrial tachycardia originating from the hepatic segment of inferior vena cava in interruption of inferior vena cava with azygos continuation. J Interv Card Electrophysiol. 2013;36:261-6.

10. van der Horst RL, Hastreiter AR. Congenital interruption of the inferior vena cava. Chest. 1981;80:638-40.

11. Guardado FJ, Byrd TM, Petersen WG. Azygous continuation of the inferior vena cava with anomalous hepatic vein drainage. Am J Med Sci. 2012;343:259-61.

12. Schatz IJ, Fine G. Venous aneurysms. N Engl J Med. 1962;266:1310-2.

13. Agnoletti $G$, Annecchino F, Preda L, Borghi A. Persistence of the left superior caval vein: can it potentiate obstructive lesions of the left ventricle? Cardiol Young. 1999;9:285-90.

\section{Submit your next manuscript to BioMed Central and we will help you at every step:}

- We accept pre-submission inquiries

- Our selector tool helps you to find the most relevant journal

- We provide round the clock customer support

- Convenient online submission

- Thorough peer review

- Inclusion in PubMed and all major indexing services

- Maximum visibility for your research

Submit your manuscript at www.biomedcentral.com/submit 